\title{
THE IMPACT OF INFRARED ARRAY TECHNOLOGY ON ASTRONOMY
}

\author{
Giovanni G. Fazio \\ Harvard-Smithsonian Center for Astrophysics
}

\begin{abstract}
Over the past ten years a technological revolution has occurred in the development of two-dimensional infrared array detectors for astronomical observations. The wide application of these arrays for both ground-based and space observations has resulted in a profound change in the capabilities and perspective for infrared astronomy, resulting in new views of the infrared sky. A review will be presented describing these detectors, the numerous advantages they provide for astronomical observations, the present state of array technology, and the potential for future growth.
\end{abstract}

